\title{
Invertibility of the density-density response function at complex frequencies
}

\author{
Tai Kai Ng \\ Department of Physics, Massachusetts Institute of Technology, Cambridge, Massachusetts 02139
}

(Received 6 March 1989; revised manuscript received 7 August 1989)

\begin{abstract}
It is shown that there exists a one-to-one mapping between the external potential $v(r, \omega)$ and induced density fluctuation $n(r, \omega)$ for complex frequencies $\omega(\operatorname{Im} \omega \neq 0)$ in the linear-response regime, for a system of electrons which satisfies the Schrödinger equation. We point out different ways where this result can be used to construct Kohn-Sham-type schemes for calculation of the density-density response function at finite (real) frequencies.
\end{abstract}

It has been pointed out by $\mathrm{Ng}$ and $\mathrm{Singwi}^{1}$ (NS) that there exists a one-to-one mapping between the applied external potential $v(r, \omega)$ and the induced density fluctuation $n(r, \omega)$ in the linear-response regime, for a system of electrons which satisfies the second law of thermodynamics $^{1}$ (NS theorem). However, this result was criticized by Gross, Mearns, and Oliveira, ${ }^{2}$ who demonstrate by using an explicit example that this result is not necessarily applicable to systems which satisfy the Schrödinger equation only. Of course, this conclusion is not surprising since it is well known that the Schrödinger equation is not really compatible with the second law of thermodynamics ${ }^{2}$ (the Schrödinger equation is time reversible, whereas the second law of thermodynamics implies time irreversibility). However, their result does raise the question about the usefulness of the NS theorem, since in practical calculations (i.e., the Kohn-Sham theory ${ }^{3}$ ), it is always assumed that the system under consideration obeys the Schrödinger equation only. The NS theorem is not applicable in this situation and thus its usefulness is questionable.

We shall address this problem in this Rapid Communication. We shall show that the NS theorem can be made applicable by using a trick in the process of calculation, by introducing an imaginary part to the frequency variable, i.e., by letting $\omega \rightarrow \omega+i \delta$ in the (Kohn-Sham) response function, where $\delta \neq 0$.

Let $\Delta W$ be the work done on the system by the external potential (to second order of the external potential). We shall prove that $\Delta W>0$ for applied potentials with frequencies $\omega \neq 0$ if in the density-density response function we let $\omega \rightarrow \omega+i \delta$, where $\delta>0$.

The work done (to second order of the external poten- tial) is given by ${ }^{1,4}$

$\Delta W=\int \frac{d \omega}{2 \pi} \int d^{3} r \int d^{3} r^{\prime} \omega v^{*}(\mathbf{r}, \omega) \chi^{\prime \prime}\left(\mathbf{r}, \mathbf{r}^{\prime}, \omega\right) v\left(\mathbf{r}^{\prime}, \omega\right)$,

where $\chi^{\prime \prime}\left(\mathbf{r}, \mathbf{r}^{\prime}, \omega\right)$ is the imaginary part of the densitydensity response function $\chi\left(\mathbf{r}, \mathbf{r}^{\prime}, \omega\right)$.

In the spectral representation for $\chi$, we have

$$
\begin{aligned}
\chi\left(\mathbf{r}, \mathbf{r}^{\prime}, \omega\right)=\sum_{m ; n} & \left(e^{-\beta E_{m}}-e^{-\beta E_{n}}\right) \\
& \times \frac{\langle n|n(\mathbf{r})| m\rangle\left\langle m\left|n\left(\mathbf{r}^{\prime}\right)\right| n\right\rangle}{\omega+E_{n}-E_{m}+i \delta},
\end{aligned}
$$

where $|n\rangle,|m\rangle$ are eigenstates of the system with energies $E_{n}$ and $E_{m}$, respectively, and $\beta=\left(k_{B} T\right)^{-1}$. The imaginary part of the response function is given by

$$
\begin{aligned}
\chi^{\prime \prime}\left(\mathbf{r}, \mathbf{r}^{\prime}, \omega\right)=\sum_{m ; n} & \frac{-\delta\left(e^{-\beta E_{m}}-e^{-\beta E_{n}}\right)}{\left(\omega+E_{n}-E_{m}\right)^{2}+\delta^{2}} \\
& \times\langle n|n(\mathbf{r})| m\rangle\left\langle m\left|n\left(\mathbf{r}^{\prime}\right)\right| n\right\rangle,
\end{aligned}
$$

for $\delta \neq 0$.

With Eqs. (1) and (3), we get

$$
\Delta W=\int \omega \frac{d \omega}{\pi} \sum_{m ; n} \frac{-\delta\left(e^{-\beta E_{m}}-e^{-\beta E_{n}}\right)}{\left(\omega+E_{n}-E_{m}\right)^{2}+\delta^{2}}\left|V_{m n}\right|^{2},
$$

where

$$
V_{m n}=\int d^{3} r v(\mathbf{r}, \omega)\langle m|n(\mathrm{r})| n\rangle .
$$

Equation (4) can be rewritten after some simple algebra as

$$
\Delta W=\int \frac{d \omega}{\pi} \sum_{E_{m}>E_{n}} \frac{4 \delta \omega^{2}\left(E_{m}-E_{n}\right)\left(e^{-\beta E_{n}}-e^{-\beta E_{m}}\right)}{\left[\left(\omega+E_{n}-E_{m}\right)^{2}+\delta^{2}\right]\left[\left(\omega+E_{m}-E_{n}\right)^{2}+\delta^{2}\right]}\left|V_{m n}\right|^{2},
$$

where $\Delta W>0$ for $\omega \neq 0$ if (i) $\delta>0$ and (ii) $V_{m n} \neq 0$ for some states $m, n$.

However, the second condition is always satisfied because the external perturbation

$$
H^{\prime}=\int d^{3} r d t v(\mathbf{r}, t) n(\mathrm{r}, t)
$$

does not commute with the Hamiltonian of the system (except the trivial case $v=$ const), thus proving our statement.

Now following $\mathrm{Ng}$ and Singwi, ${ }^{1}$ the statement $\Delta W>0$ for all (nontrivial) external potentials $v$ (to second order in $v$ ) implies that there exists a one-to-one mapping between the external potential $v(r, \omega)$ and the induced density fluctuation $n(r, \omega)$, in the linear-response regime. Thus a density-functional theory can be established in this 
case. In particular, a Kohn-Sham type of theory can be formulated (see, for example, Refs. 5 and 6) since we have used only the Schrödinger equation in our proof.

Physically, the introduction of a finite imaginary part in the frequency variable couples the system phenomenologically to an external heat bath, thus enforcing the second law of thermodynamics $(\Delta W>0)$ on the system and making the NS theorem applicable. It is only in the limit $\delta$ $\rightarrow 0$, when the system decouples from the heat bath, that the NS theorem is not applicable. Alternatively, we can also view our approach as an analytical continuation of the frequency variable $\omega$ onto the complex $\omega$ plane, and our result shows that a density-functional theory can be established as long as $\omega$ is complex. It is only in the limit when $\omega$ becomes real that the existence theorem breaks down. (For $\delta<0$ and $\Delta W<0$ the existence theorem still holds. The theorem breaks down only when $\Delta W=0$ for some external potentials.)

Now we shall discuss the consequences of the theorem. A natural consequence of the theorem is that we can now formulate a Kohn-Sham type of theory for the densitydensity response function $\chi\left(r, \mathbf{r}^{\prime}, \omega+i \delta\right)$ for any complex frequencies $\omega+i \delta(\delta>0)$ for the system of interest, ${ }^{5,6}$ and the physical response function $\chi_{\text {ret }}\left(r, \mathbf{r}^{\prime}, \omega\right)$ can be obtained by analytic continuation (extrapolation) of $\chi\left(\mathbf{r}, \mathbf{r}^{\prime}, \omega+i \delta\right)$ to the $\delta \rightarrow 0$ limit [by, for example, computing $\chi\left(\mathbf{r}, \mathbf{r}^{\prime}, \omega\right.$ $+i \delta)$ for several decreasing values of $\delta$ 's $(\delta>0)$ ]. Notice that this analytic continuation procedure is actually the standard one of which a retarded response function is being defined. Thus the continuation gives the correct retard response function as long as the standard definition of the retarded response function remains valid. It is important to keep in mind that this analytic continuation procedure is defined for $\chi$ only, but not for the inverse function $\chi^{-1}$, since $\chi^{-1}$ may not be well defined in the $\delta \rightarrow 0$ limit. (The existence theorem breaks down in the limit $\delta \rightarrow 0$. ) However, this procedure suffers from the technical difficulty that the exchange-correlation potential $\phi_{\mathrm{xc}}(r, \omega)$ thus defined ${ }^{5,6}$ may become singular in the limit $\delta \rightarrow 0$. This may make the calculation difficult in practice as $\delta$ becomes small. To overcome this difficulty, we sug-

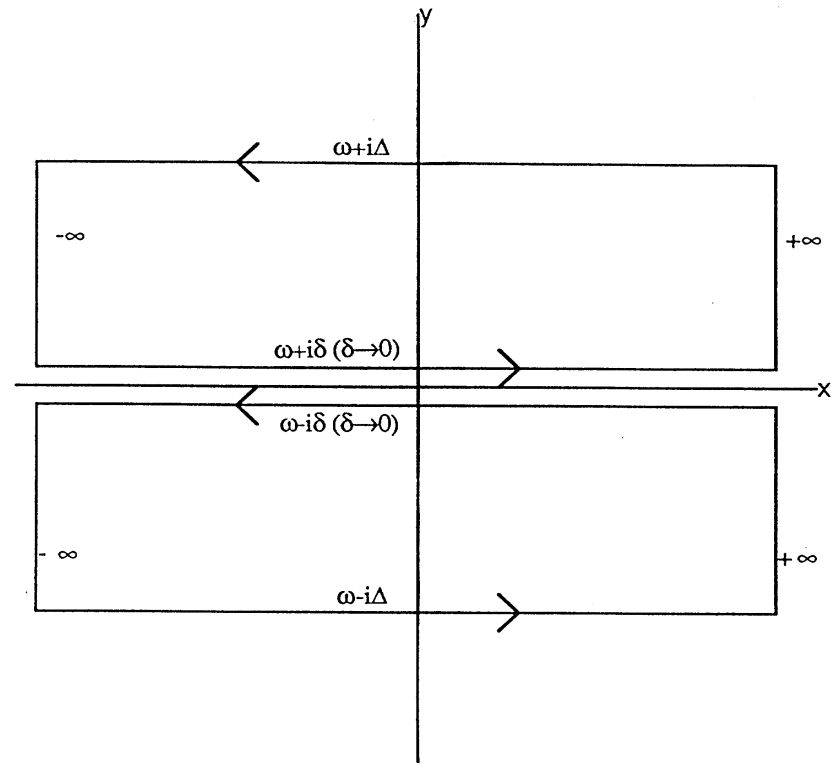

FIG. 1. Contour in the complex $z$ plane in Eq. (8).

gest the following alternative procedure.

First of all, we observe that any response function $\chi(\mathbf{r}$, $\mathbf{r}^{\prime} ; z$ ) ( $z$ denotes the complex frequency) can be written in the spectral representation as (we shall omit the variables $\mathbf{r}, \mathbf{r}^{\prime}$ in the following for brevity)

$$
\chi(z)=\int_{-\infty}^{+\infty} \frac{A(\omega)}{z-\omega} d \omega,
$$

where $A(\omega)$ is the spectral function. Thus $\chi(z)$ has singularity (pole plus branch cut) only on the real axis.

Now we construct the contour integral $I$ as shown in Fig. 1 where

$$
I=\int_{C} \chi(z) f(z) d z=0,
$$

for any function $f(z)$ which is analytic inside the contour (and on the real axis). Thus we have

$$
\int_{-\infty}^{+\infty} \chi(\omega-i \Delta) f(\omega-i \Delta) d \omega-\int_{-\infty}^{+\infty} \chi(\omega+i \Delta) f(\omega+i \Delta) d \omega=2 \pi i \int_{-\infty}^{+\infty} f(\omega) A(\omega) d \omega
$$

In particular, if we let $f(z)=e^{i z t}$, then Eq. (9) gives the Fourier transform of the spectral function $A(t) . A(\omega)$ can be determined by Fourier transforming back and $\chi(z)$ (for any $z$ ) can be determined by using Eq. (7). Thus a Kohn-Sham-type scheme can be used to compute the density-density response function $\chi\left(\mathbf{r}, \mathbf{r}^{\prime} ; z\right)$ for $z=\omega \pm i \Delta$ $(\Delta \neq 0)$ for all $\omega$, and the physical response function $\chi_{\text {ret }}\left(\mathbf{r}, \mathbf{r}^{\prime}, \omega\right)$ can be obtained by using steps as described above. Notice that this procedure involves only complex frequencies in the Kohn-Sham scheme where $\chi^{-1}$ is always well behaved. Thus the technical difficulty in taking the $\delta \rightarrow 0$ limit is always being avoided.

It should also be pointed out that the above discussion is not restricted to the density-density response function only. In particular, a similar statement can be made for the current-current response function, leading to the possible formulation of a current-functional theory in complex frequency describing the linear responses of electronic systems to arbitrary form of electromagnetic fields. ${ }^{6}$

In summary, we show in this Rapid Communication that it is possible to formulate a density-functional theory in the linear-response regime, of which a Kohn-Sham approach can be constructed, provided that the frequency variable is complex. The density-density response function $\chi\left(r, \mathbf{r}^{\prime}, \omega+i \delta\right)$ can be computed and the physical results can be obtained by well-defined mathematical methods.

This work is supported by the National Science Foundation under Grant No. DMR-8521377. 
${ }^{1}$ T. K. Ng and K. S. Singwi, Phys. Rev. Lett. 59, 2630 (1987).

${ }^{2}$ E. K. U. Gross, D. Mearns, and L. N. Oliveira, Phys. Rev. Lett. 61, 1518 (1988); see also T. K. Ng and K. S. Singwi, ibid. 61, 1519 (1988).

${ }^{3}$ W. Kohn and L. J. Sham, Phys. Rev. 140, A1133 (1965).
${ }^{4}$ See for example, D. Forster, Hydrodynamic Fluctuations, Broken Symmetry, and Correlation Functions (Benjamin, New York, 1983).

${ }^{5}$ E. K. U. Gross and W. Kohn, Phys. Rev. Lett. 55, 2850 (1985).

${ }^{6}$ T. K. Ng, Phys. Rev. Lett. 62, 2417 (1989). 